\title{
Entre_Cabanyal upgrade. Versiones y actualizaciones en las obras de arte digital interactivo
}

\author{
Trinidad Gracia Bensa ${ }^{1}$; Emilio Martínez Arroyo ${ }^{1}$; Emanuele Mazza ${ }^{1}$ \\ ${ }^{1}$ Laboratorio de Luz, Universitat Politècnica de València \\ tgracia@esc.upv.es \\ ejmartinez@esc.upv.es \\ emanuelemazza@d3cod3.org
}

\begin{abstract}
In this article we are going to analyze some issues related to interactive digital artworks preservation. The main problems are the electronic components damage and obsolescence of both software and hardware, the new operating systems incompatibility and software obsolescence with which the artwork was made.

Artists have had to look for appropriate preservation strategies, because after a few years the artworks can no longer be reassembled as they were originally produced, applying solutions such as emulation or upgrading to new work versions. These decisions uncover some questions about what the most convenient thing is to do when a work cannot be reinstalled again because of the deterioration of the computer components. When remaking these works, can we consider them as versions or as new works?
\end{abstract}

We will focus the communication on these issues from both a theoretical and a practical point of view, discussing what this rapid deterioration of interactive digital works implies and also how to document these works in order to be able to reproduce them in the future.

Specifically, we will describe the steps followed in the new version of the interactive audiovisual installation Entre_Cabanyal, made in 2002, originally programmed with Macromedia Director 8.5 software, on a Windows 2000 PC, and a Miro DC30 video capture card. We expose the reasons that have led us to reprogram the work with $\mathrm{C++}$ language, considering that the use of proprietary software would probably limit the maintenance of the work in the future, and under Linux operating system, also open source, since both Windows and Mac are increasing incompatibilities in their new operating systems.

Keywords: digital art, interactivity, obsolescence, upgrade, preservation. 
Entre_Cabanyal upgrade. Versiones y actualizaciones en las obras de arte digital interactivo

\section{Resumen}

En este artículo vamos a analizar algunas cuestiones vinculadas a la preservación de las obras digitales interactivas. Los principales problemas devienen del deterioro de los componentes y obsolescencia tanto del software como del hardware, la incompatibilidad de los nuevos sistemas operativos y que los programas informáticos con los que se hicieron quedan también obsoletos.

Los artistas hemos tenido que buscar estrategias de preservación adecuadas porque tras pocos años las obras ya no se pueden volver a montar como se produjeron originalmente, aplicando soluciones como la emulación o la actualización de nuevas versiones de la obra. Estas decisiones destapan algunas cuestiones al respecto sobre qué es lo más conveniente cuando una obra no puede volver a instalarse de nuevo por el deterioro de los componentes informáticos. ¿Al actualizar estas obras podemos considerarlas como versiones o como nuevas obras?

La comunicación se centrará en estas cuestiones desde el punto de vista teórico y también práctico, analizando lo que supone este deterioro tan rápido de las obras digitales interactivas y también cómo documentarlas para poder reproducirlas en un futuro.

En concreto describiremos los pasos seguidos en la nueva versión de la instalación audiovisual interactiva Entre_Cabanyal, realizada en 2002, originalmente programada con el software Macromedia Director 8.5, en un ordenador PC con Windows 2000 y una capturadora de video Miro DC30. Exponemos los motivos que nos han llevado a volver a programar la obra con lenguaje $\mathrm{C}++$, por considerar que el uso de software propietario previsiblemente limitaría más en un futuro su mantenimiento, y bajo el sistema operativo Linux, también open source, dado que tanto Windows como Mac están incrementando las incompatibilidades en sus nuevos sistemas operativos.

Palabras clave: arte digital, interactividad, obsolescencia, actualización, preservación. 


\section{Introducción}

Centrar nuestro trabajo en el arte digital nos hizo sentir estábamos en una ruta de futuro, y también que el medio aportaba al trabajo unas características que rompían con la tradición académica: la posibilidad de hacer copias idénticas cuestionaba la unicidad de la obra; transmitirla por la red reforzaba cierto carácter inmaterial, y además pensábamos que se podía almacenar de forma segura y económica. Pero los medios digitales han avanzado tan rápido que los soportes de las obras se han quedado obsoletos, antiguos, y fuera de esa idea de futuro que las motivó.

Consideramos que es importante conservar la obra en su forma y formato originales, siempre que sea posible; y cuando ya no lo es, buscar la mejor estrategia para que la obra mantenga su significación y se despliegue de la forma más semejante, adaptando los dispositivos y lenguaje de programación a los sistemas que ofrezcan más posibilidades de durabilidad. Vamos a centrar el análisis de todos estos aspectos en el proceso seguido para la actualización de la obra del Laboratorio de Luz, Entre_Cabanyal.

Si a finales del siglo XX se produjo un desplazamiento del valor simbólico entre el objeto y el concepto en la obra de arte (Marchán Fiz, 1994), en el arte digital ese desplazamiento se produce entre el objeto y la información. En este sentido, desde el campo de la restauración se contempla también ese cambio respecto a la preservación de las obras de arte digital: "Si el objeto carece de valor simbólico relevante, y lo importante es la información que soporta, entonces el objeto en sí es sacrificable en tanto se conserve su información" (Muñoz Viñas, 2010, p. 73), contemplando el duplicado o transferencia de los datos a otro soporte.

Pero esa transferencia de datos conlleva matices. Podemos verla de un modo más directo en el caso de obras de net.art por su carácter inmaterial y de puro lenguaje de programación, pero incluso aquí el software y/o el lenguaje de programación utilizado pueden haberse quedado obsoletos o ser incompatibles con los navegadores o los sistemas operativos actuales, como sucede en las obras realizadas con Flash y actionscript, o las que funcionan con ventanas emergentes.

En el caso de instalaciones de arte interactivo, los matices se multiplican, al incrementarse también los tipos de dispositivos digitales o electrónicos, los objetos físicos que pueden intervenir, así como su relación con el espacio y los espectadores o espectadoras. Aquí la actualización de la obra debe preservar la información digital y las características simbólicas y conceptuales de su configuración espacial, por eso presenta mayores retos en su preservación y es importante encontrar la estrategia adecuada. Esperamos que el proceso seguido para la actualización de la obra Entre_Cabanyal cumpla estas expectativas. 


\section{Entre_Cabanyal}

Entre_Cabanyal se realizó en 2002 para la 15a exposición audiovisual que la Facultad de Bellas Artes de Bilbao organizaba en la Fundación BBVA cada año con una selección de obras de sus estudiantes junto a proyectos de artistas invitados. Quisimos aprovechar esta oportunidad para mostrar en el País Vasco la problemática social que en aquellos años se estaba dando en el Barrio del Cabanyal de Valencia, debida al proyecto de ampliación de una avenida que dividiría por dos el barrio, proyecto que finalmente quedó anulado el año 2016, después de 18 años de lucha de los vecinos (Plataforma Salvem el Cabanyal, s. f.).

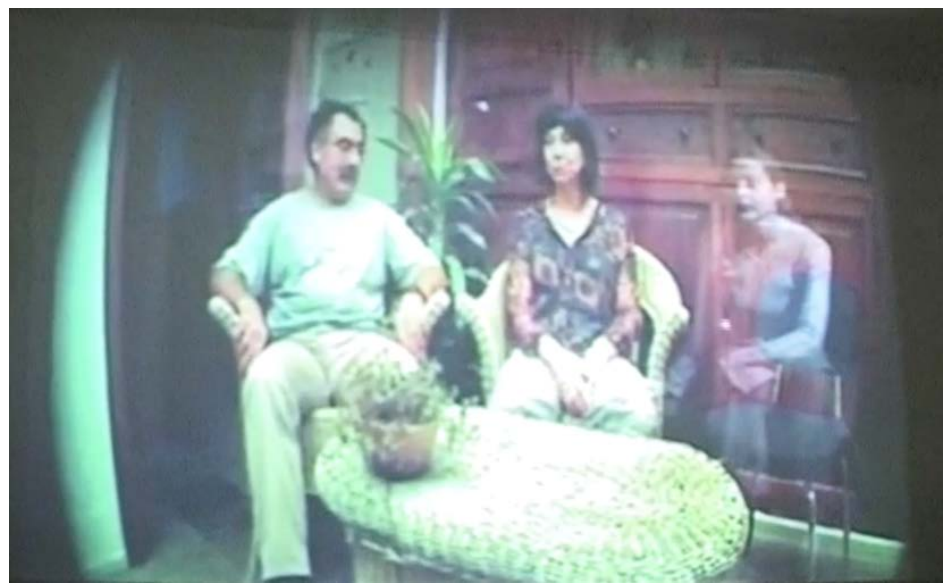

Fig. 1. Entre_Cabanyal. Cabanyal Portes Obertes (2005). Proyección en zona 2

En el año 2003, formó parte del Festival Escena Contemporánea organizado por Llorenç Barber en el Círculo de Bellas Artes de Madrid y en 2005 se expuso en el Barrio del Cabanyal, dentro de VIII Edición Cabanyal Portes Obertes. El 2 de julio de 2021 se ha presentado la nueva versión en la exposición 1998-2015 Cabanyal Portes Obertes. Cultura y ciudadanía en el Centro de Cultura Contemporánea del Carmen de Valencia, por lo que hemos podido recoger en este artículo todos lo pasos seguidos en su actualización. 


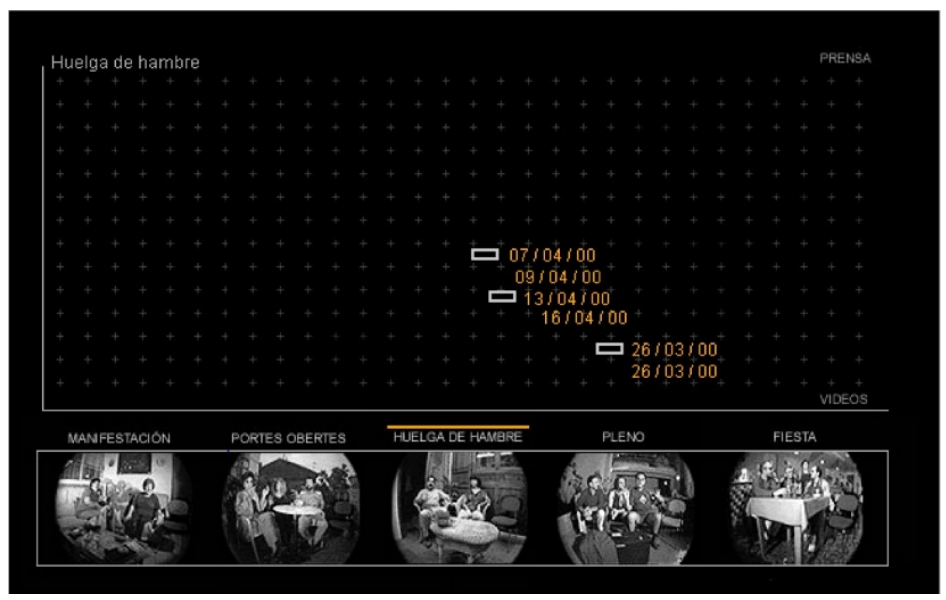

Fig 2. Entre_Cabanyal (2002). Interface proyectada en zona 1

Casi 20 años en la línea temporal del desarrollo tecnológico es mucho tiempo, por lo que la estrategia a seguir para la actualización de la pieza ha sido un poco radical. Lo deseable es hacer pequeñas actualizaciones periódicas: "the refreshing, which consists in the periodic transference of the digital information of a media in danger of obsolescence to a better adapted media" (García \& Montero Vilar, 2010, p. 48). Lejos de la posibilidad de refrescar toda la información y dispositivos de la obra, hemos tenido que sustituir el hardware, reinterpretar o traducir el código de programación, preservar gran parte de los contenidos originales (archivos de imagen), y migrar los archivos de vídeo a un nuevo códec.

\subsection{Planteamiento de la obra}

"Con el objetivo de involucrar al espectador hemos planteado un sistema de circuito cerrado que introduce su imagen en la escena, ocupando una silla vacante que aparece en las cinco conversaciones que grabamos con vecinos del barrio, en las que nos cuentan los actos de resistencia que han realizado para defender sus casas, su modo de vida". (Laboratorio de Luz, s. f.)

La instalación se articula de dos zonas contiguas. La zona 1 actúa a modo de cabina de proyección, desde ahí, un/a visitante (A) sentado/a en un banco de color negro, puede seleccionar con el ratón, en una interface gráfica que visualiza a través de un monitor, una de las 5 conversaciones pregrabadas. Al mismo tiempo, su imagen es registrada en tiempo real por una cámara de video, y superpuesta en la proyección que se presenta 
en la zona 2, espacio de proyección donde los demás espectadores y el mismo ven la fusión del video y la imagen de A, tal como señala la cita anterior: "ocupando una silla vacante que aparece en las cinco conversaciones". Desde la interface de la zona 1, y mientras se reproduce el video, el/la visitante A puede encontrar información adicional sobre lo que le están comentando los vecinos.

La idea de entrar en la pantalla es un clásico del cine. En la película Sherlock Jr. (1924), Buster Keaton sueña que salta a la pantalla y se integra/no-integra con los cambios de plano que allí acontecen. No todo encaja, la realidad de las cosas que pasan o los deseos proyectados -que, en nuestra obra, aluden a la problemática del barrio del Cabanyal-señalan una situación que amenazaba con dejarnos/dejarles fuera de lugar.

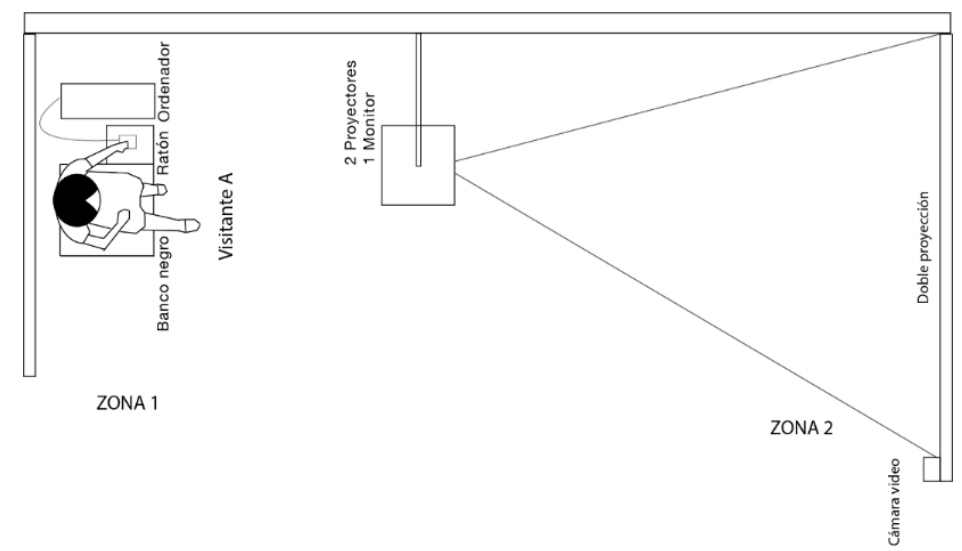

Fig 3 Plano de la instalación en 2021
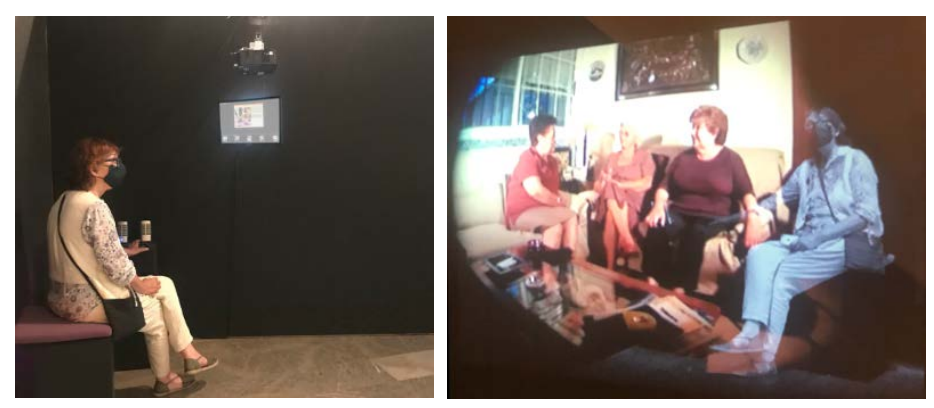

Fig 4. Zona 1 y zona 2 de la instalación, 2021 


\subsection{Planteamiento de la actualización}

Las cualidades estéticas y significativas de la instalación no dependen de la utilización de una tecnología específica, si bien, esos dispositivos gestionan la representación del contenido y posibilitan la interactividad con el/la visitante por lo que le aportan su lógica a través del funcionamiento de su interface.

A nivel artístico consideramos que el aspecto más destacado del sistema de interacción en esta obra es la fusión de la imagen del espectador o de la espectadora con la de los vecinos y vecinas para que interactúe en su conversación, pero a nivel de la preservación de la obra hemos de poner el foco en la traducción o reinterpretación del sistema técnico y el código original a una nueva plataforma y lenguaje.

La traducción no se encuentra, como la poesía, en el propio interior del bosque agreste de la lengua, por decirlo así, sino que, desde fuera de ella, enfrente de ella, y sin entrar en ella, llama al original a entrar, y a entrar en aquel único sitio donde el eco respectivo en la propia lengua puede dar la resonancia de una obra en otra. (Benjamin, 1996, p. 342)

Aunque el contexto del código parece muy lejano al de la poesía, la traducción de un lenguaje de programación a otro también implica encontrar las resonancias del lenguaje para que la obra original resuene de la forma más semejante posible en la actualizada.
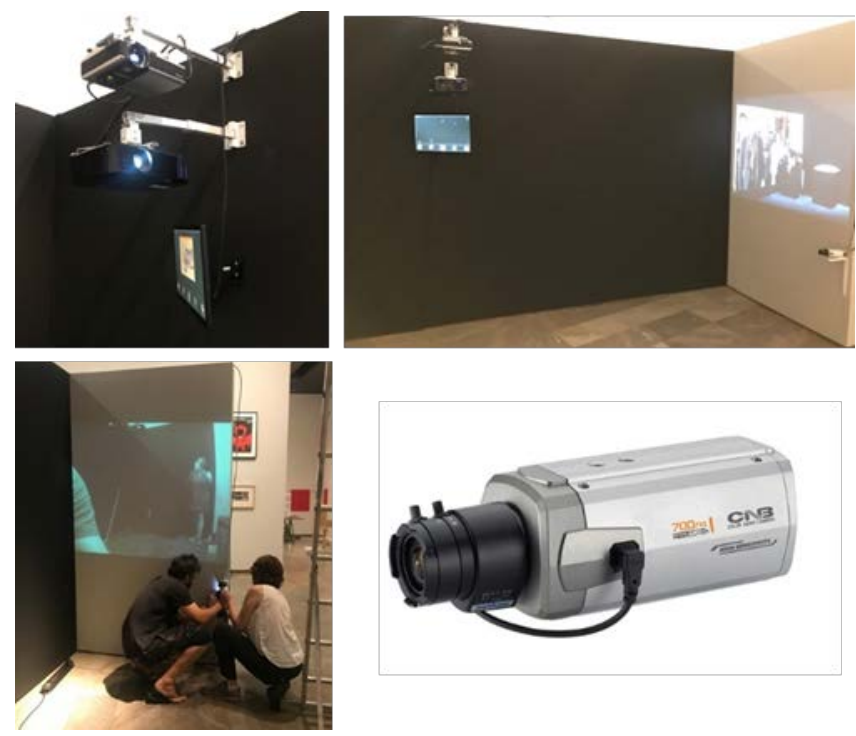

Fig 5. Monitor con la interface gráfica, proyectores de video, cámara CCTV y proceso de montaje, 2021 
Entre_Cabanyal upgrade. Versiones y actualizaciones en las obras de arte digital interactivo

Parte del hardware y software original no eran elementos que aportarán una determinada estética a la instalación, sin embargo, otros sí transmitían los rasgos epocales, como la cámara analógica CCTV, los proyectores de video, así como el diseño de la interfaz en la que se ha mantenido la tipografía y la composición de imágenes. La caja de la CPU y el ratón utilizados no tienen un diseño destacado por lo que su sustitución no afecta de forma directa a la obra. Lo que si afecta a la experiencia de los espectadores y las espectadoras es la lógica de funcionamiento y es importante mantenerla igual.

\section{Estrategias de preservación}

Las decisiones tomadas para actualizar la obra Entre_Cabanyal han pasado por un proceso híbrido en el que se han tenido en cuenta cuestiones de:

- Conservación digital, que nos impulsó a documentar bien la obra original y todos los procedimientos aplicados en su actualización.

- Cultura del ordenador y tecnología de los media, analizamos las cualidades del hardware y software original para mantener, en la medida de lo posible los formatos de archivo y estructura de datos de los contenidos.

- Teoría y cultura de los media, analizamos la obra desde el punto de vista de la historia y ética de los medios, para valorarla en función de sus características históricas, artísticas y culturales.

La primera estrategia de preservación que se recomienda es la emulación. Ciertamente se podía emular en un ordenador actual un sistema operativo que permitiera la aplicación de Macromedia Director. También es posible encontrar en las plataformas de segunda mano el periférico que utilizaba. Pero al analizar la obra desde las cuestiones antes señaladas, y dado que somos sus autores, descartamos esta opción en pro de conseguir una mayor durabilidad, pensando también en un futuro en el que ya no podamos estar.

Otra de las estrategias seguida es la migración, aun teniendo en cuenta que puede conllevar pérdida de calidad: "The degeneration or loss of quality increases exponentially with the migration generation. A migration of third or fourth generation doesn't prob- ably satisfy the minimum quality required by the artist." (García \& Montero Vilar, 2010, p. 50). Pese a ser conscientes de esa posible pérdida, hemos tenido que utilizarla en los cinco archivos de video, tal y como se detalla en los siguientes apartados.

La última estrategia utilizada es la reinterpretación del código a otro lenguaje y también la sustitución del hardware, un nuevo ordenador con otro sistema operativo de base: 
"The assurance or approval of the artist, but it may be the only way to guarantee the re-creation, installation, or re-design of the artwork" (García \& Montero Vilar, 2010, p. 51).

Como se detalla en los siguientes apartados hemos realizado tanto la migración de algunos de los contenidos como la reinterpretación de todos los componentes físicos de la instalación.

\subsubsection{Elección del sistema operativo}

La versión original estaba programada en una plataforma Windows 2000, pero a la hora de migrar la obra se eligió el sistema operativo open source Linux en su distro Ubuntu 21.04 .

Esta decisión se vio ratificada cuando leímos que, ante la migración de una de las obras emblemáticas del arte interactivo, Legible City (1989) de Jeffrey Shaw, Bernhard Serexhe, curador principal del ZKM, respecto a la elección del sistema operativo señaló:

Linux was selected as the target platform for the porting. Linux is a free Unix variant that runs on the most diverse hardware platforms -including on Apple computerswhich is constantly being adapted to new hardware, and, current expectations are that due to its origins [Unix], it will have a long lifespan. (Copestake, 2013)

El caso de estudio de la actualización de Legible city por parte del proyecto Digital art Conservation "Digital Art Conservation», (s. f.) ha sido de gran ayuda a la hora de tomar decisiones, pues se trata de una instalación interactiva originalmente programada con un software propietario y con un hardware obsoleto: Indigo 2 de Silicon Graphic, y almacenar equipos semejantes suponía un alto coste para el museo y no garantizaba la perdurabilidad de la obra, por ello decidieron migrarla a un sistema Linux.

Para Entre_Cabanyal hemos elegido el sistema operativo Ubuntu 21.04. Ubuntu es una distribución de Linux basada en Debian. Como la placa base de la nueva máquina es ASUS Tuf Gaming B560M-Plus y el procesador Intel Core i7 10700 BOX, la versión utilizada de Ubuntu es la 21.04, última versión estable del sistema y que es la única compatible con las características de la placa base de ese ordenador.

\subsubsection{Traducción a otro lenguaje de programación}

En el año 2002 se programó la obra con lenguaje Lingo, un lenguaje de programación orientado a objetos que venía incorporado con el software propietario Macromedia Director. 
Lingo fue desarrollado por John H. Thompson en 1989 para Macromind Director, software que en 1993 se convirtió en Macromedia Director. Durante la década de 1990 e inicios del 2000 fue uno de los lenguajes para productos multimedia interactivos más utilizado. En el año 2008, la compañía Abobe adquirió el software de Macromedia y en 2017 anunció su final.

A la hora de elegir el nuevo lenguaje para traducir el código se optó por desarrollar una aplicación específica programada en $\mathrm{C}++$, basada en openFrameworks.

openFrameworks es un entorno de programación open source en C++ desarrollado inicialmente por Zachary Lieberman en 2005 (OF v0.01). Su mantenimiento y desarrollo ha sido posible gracias a Zachary Lieberman, Theo Watson y Arturo Castro con contribuciones de otros miembros de la comunidad que se ha ido generando con el tiempo, aportando una amplia base de librerías para que artistas y diseñadores desarrollen aplicaciones interactivas (Noble, 2009, p. 153).

La filosofía y lenguaje de ambos entornos de programación es muy diferente. Director establecía una metáfora cinematográfica, como si el desarrollador fuera el director de cine. La ventana de salida se denominaba Stage, a modo de escenario donde se reproducían todos los elementos de la aplicación; estos elementos podían ser gráficos, texto, video o audio, se organizaban en la ventana del Cast y se denominaban Cast membres. El orden temporal de aparición se organizaba en la ventana de la línea de tiempo denominada Score, en la que se podían establecer diferentes escenas. La disposición de los sprite en el score y su ubicación en el stage eran la base del sistema de reproducción de la película en Director.

La lógica de trabajo en openFrameworks es muy diferente, el entorno de programación aporta una serie de clases y métodos para programar la disposición y comportamiento de gráficos, texto, video, audio..., articulando la estructura de programación en tres bloques principales: setup, update y draw. Es un sistema de programación creativa cuya estructura se asemeja al de Processing, pero sobre la base de C++ y no de Java.

Debido a estas diferencias, la traducción del código sigue la lógica del funcionamiento de la obra, como capa invisible que hace resonar la obra de forma semejante a la original, aunque no siga aquella estructura del código Lingo.

\subsubsection{Cambios de percepción en la imagen}

La percepción de la imagen en el arte digital depende de su resolución y de las características del dispositivo que la acoge; cuando este dispositivo ha quedado obsoleto, es habitual que las características del nuevo sean diferentes. La interface original tenía un formato $4: 3$ con resolución $1024 \times 768$, lo que ha tenido que adaptarse al nuevo formato $16: 9$ con resolución 1280 x 720. Los iconos para activar la 
reproducción de los videos tenían un tamaño de 122×122 pixeles, como estaban hechos a partir de un frame de cada uno de los videos, se ha reproducido su diseño con un tamaño de $180 \times 180$ píxeles.

Para el tratamiento de los archivos de video se conjuga las estrategias de preservación del video arte y del arte interactivo. Algunos expertos recomiendan el códec de video Motion JPEG2000 y el contenedor MXF (Material Exchange Format) como formato de preservación para el vídeo digital (Escobedo, 2011) (Venturini \& Santi, 2013, p. 217), o en cualquier caso tender hacia formatos sin pérdidas. Cuando se hizo la obra, las cuestiones de preservación del video estaban más orientadas a los formatos de cinta que a los archivos digitales, por lo que no se tuvieron en cuenta estas consideraciones.

El hecho de que la capturadora Miro DC30 reprodujera el archivo de video por su salida analógica, cuando era seleccionado desde la aplicación de Director, lo vimos como una facilidad técnica, pero a la hora de actualizar la obra ha significado una dificultad añadida. Porque este hecho solo se producía si los videos tenían su códec propietario. La única manera que hemos encontrado para exportar esos archivos de video ha sido con FFmpeg a través de la terminal, frame a frame, manteniendo su formato original DV PAL 720 x 576 píxeles cuadrados, sin campos, y con el códec JPEG 2000 tal como recomiendan por tener menos pérdida de cara a futuras adaptaciones.

\subsection{Ficha técnica comparativa}

El esquema que sigue la información de este apartado se basa en el documento Media Elements for Computer-Based Artworks (Matters in Media Art, 2015), y en él se comparan los datos técnicos originales de la obra y los de su actualización en 2021.

\begin{tabular}{|c|c|}
\hline Versión 2002 & Versión 2021 \\
\hline \multicolumn{2}{|c|}{ 1. INFORMACIÓN GENERAL } \\
\hline \multicolumn{2}{|l|}{ Parámetros de presentación } \\
\hline \multicolumn{2}{|c|}{$\begin{array}{l}\text { Instalación audiovisual interactiva para un espacio interior dividido en dos zonas. La } \\
\text { zona } 1 \text { actúa a modo de cabina de proyección para un/a visitante. La zona } 2 \text { para } \\
\text { los/as demás visitantes. Misma distribución en ambas versiones. }\end{array}$} \\
\hline \multicolumn{2}{|c|}{ Interacción / participación de los visitantes } \\
\hline \multicolumn{2}{|c|}{$\begin{array}{l}\text { Interactividad mediante ratón para un solo visitante en la zona } 1 \text {, quien además } \\
\text { participa con su imagen en la doble proyección de la zona } 2 .\end{array}$} \\
\hline \multicolumn{2}{|l|}{ Estructura } \\
\hline Dispositivos y código propietarios. & Dispositivos y código abierto. \\
\hline
\end{tabular}


Entre_Cabanyal upgrade. Versiones y actualizaciones en las obras de arte digital interactivo

\begin{tabular}{|c|c|}
\hline \multicolumn{2}{|l|}{ Entorno informático } \\
\hline $\begin{array}{l}\text { - Plataforma: Windows } \\
\text { - Procesador Intel Pentium } 4 \\
\text { - } 128 \mathrm{Mg} \text { RAM } \\
\text { - Periféricos: Capturadora Miro DC30 }\end{array}$ & $\begin{array}{l}\text {-Plataforma: Linux } \\
\text {-Procesador Intel Core i7 } 10700 \\
\text { BOX } \\
\text {-16 GB RAM }\end{array}$ \\
\hline \multicolumn{2}{|c|}{ 2. INFORMACIÓN SOBRE LOS COMPONENTES } \\
\hline $\begin{array}{l}\text { Número componentes: } 10 \\
\text { - } 2 \text { proyectores video XGA }(1024 \times 768) \\
\text { - } 1 \text { monitor TV Sony Triniton, 16' } \\
\text { - } 1 \text { ordenador PC sobremesa } \\
\text { - } 1 \text { capturadora video DC30 } \\
\text { - } 1 \text { mini-cámara video B/W CCTV } \\
\text { - } 1 \text { ratón de ordenador } \\
\text { - } 1 \text { soporte de } 2 \text { baldas para video } \\
\text { proyectores } \\
\text { - } 1 \text { banco/asiento negro } \\
1 \text { foco iluminación baja intensidad }\end{array}$ & $\begin{array}{l}\text { Número componentes: } 9 \\
\text { - } 1 \text { proyector video HD (1280x720) } \\
\text { entrada HDMI } \\
\text { - } 1 \text { proyector video XGA } \\
\text { (1024x768) entrada RCA } \\
\text { - } 1 \text { monitor LCD Hanns spree. } \\
21,5^{\prime \prime} \\
\text { - } 1 \text { ordenador PC sobremesa } \\
\text { - } 1 \text { cámara video Camtronic B/W } \\
\text { CCTV } \\
\text { - } 1 \text { ratón de ordenador } \\
\text { - } 2 \text { soportes para proyectores } \\
\text { - } 1 \text { banco/asiento negro }\end{array}$ \\
\hline \multicolumn{2}{|l|}{ 3. MEDIA } \\
\hline Software & \\
\hline $\begin{array}{l}\text { - Sistema operativo: Windows } \\
\text { Versión: } 2000 \\
\text { - Programa principal } \\
\text { Nombre: Director (Macromedia) } \\
\text { Versión: } 8.5 \\
\text { Ubicación: C/Archivos de programa } \\
\text { Programación: código escrito para esta } \\
\text { obra en lenguaje Lingo por Moisés Mañas y } \\
\text { Diego Díaz. } \\
\text { No es open source. } \\
\text { - Software subsidiario } \\
\text { WDM 2.0. Driver de la capturadora Miro } \\
\text { DC30 ¿ ¿Requiere password, llave o mochila? } \\
\text { Si }\end{array}$ & $\begin{array}{l}\text { - Sistema operativo: Linux, Ubuntu } \\
\text { Versión: } 21.04 \\
\text { - Programa principal } \\
\text { Nombre: Entre_Cabanyal } \\
\text { Versión: openFrameworks } 0.11 .2 \\
\text { Ubicación: /opt } \\
\text { Programación: código escrito } \\
\text { para esta obra en lenguaje C++ } \\
\text { por Emanuele Mazza. } \\
\text { Es open source } \\
\text { - Software subsidiario } \\
\text { Ninguno } \\
\text { - ¿Requiere password, llave o } \\
\text { mochila? } \\
\text { No }\end{array}$ \\
\hline
\end{tabular}




\begin{tabular}{|c|c|}
\hline \multicolumn{2}{|l|}{ Contenidos/Assets } \\
\hline $\begin{array}{l}\text { - Tipo } \\
\text { Video (.avi) DV PAL 720×576 px, } 4: 3 \\
\text { Imágenes (.jpg) } \\
\text { - Nombre archivos y ubicación } \\
\text { data/images/cerrar.jpg } \\
\text { data/images/fiestaoff.jpg } \\
\text { data/images/fiestaon.jpg } \\
\text { data/images/fondo.jpg } \\
\text { data/images/huelgaoff.jpg } \\
\text { data/images/huelgaon.jpg } \\
\text { data/images/manifaoff.jpg } \\
\text { data/images/manifaon.jpg } \\
\text { data/images/plenooff.jpg } \\
\text { data/images/plenoon.jpg } \\
\text { data/images/portesoff.jpg } \\
\text { data/images/porteson.jpg } \\
\text { data/interface/fiesta.jpg } \\
\text { data/interface/huelga.jpg } \\
\text { data/interface/pleno.jpg } \\
\text { data/interface/portes_obertes.jpg } \\
\text { data/prensa/fiesta/170601_f.jpg } \\
\text { data/prensa/fiesta/180601_f.jpg } \\
\text { data/prensa/huelga/070400_h.jpg } \\
\text { data/prensa/huelga/090400_h.jpg } \\
\text { data/prensa/prensa/huelga/130400_h.jpg } \\
\text { data/prensa/huelga/160400_h.jpg } \\
\text { data/prensa/huelga/260300_h.jpg } \\
\text { datarensa/manifestacion/011099_mani.jpg } \\
\text { dat_mifestacion/190500_mani.jpg }\end{array}$ & $\begin{array}{l}\text { - Tipo } \\
\text { Video (.mov) } 720 \times 576 \text { px, 4:3 } \\
\text { Imágenes (.jpg) } \\
\text { - Nombre archivos y ubicación } \\
\text { Se mantiene el mismo nombre y } \\
\text { ubicación, excepto en los videos } \\
\text { que tienen formato .mov }\end{array}$ \\
\hline
\end{tabular}




\begin{tabular}{|c|c|}
\hline $\begin{array}{l}\text { data/prensa/manifestacion/240401_mani.jpg } \\
\text { data/prensa/manifestacion/250998_mani.jpg } \\
\text { data/prensa/manifestacion/270401_mani.jpg } \\
\text { data/prensa/pleno/250501_pleno.jpg } \\
\text { data/prensa/pleno/260101_pleno.jpg } \\
\text { data/prensa/pleno/260102_pleno.jpg } \\
\text { data/prensa/pleno/260501_pleno.jpg } \\
\text { data/prensa/portes/040502_portes.jpg } \\
\text { data/prensa/portes/051299_portes.jpg } \\
\text { data/prensa/portes/060502_portes.jpg } \\
\text { data/prensa/portes/111298_2_portes.jpg } \\
\text { data/prensa/portes /111298_f_portes.jpg } \\
\text { data/prensa/portes/120500_2_portes.jpg } \\
\text { data/prensa/portes /120500_f_portes.jpg } \\
\text { data/prensa/portes/250501_f_portes.jpg } \\
\text { data/prensa/portes /261199_portes.jpg } \\
\text { data/videos/fiesta.avi } \\
\text { data/videos/huelga.avi } \\
\text { data/videos/manifiesta.avi } \\
\text { data/videos/nombres.avi } \\
\text { data/videos/pleno.avi } \\
\text { data/videos/portes.avi } \\
\text { • Ubicación copias } \\
\text { Archivo Laboluz en CD-ROM }\end{array}$ & $\begin{array}{l}\text { data/videos/fiesta.mov } \\
\text { data/videos/huelga.mov } \\
\text { data/videos/manifiesta.mov } \\
\text { data/videos/nombres.mov } \\
\text { data/videos/pleno.mov } \\
\text { data/videos/portes.mov } \\
\text { - Ubicación copias } \\
\text { Código: } \\
\text { https://github.com/laboluz/entre_cab } \\
\text { anyal } \\
\text { Assets: } \\
\text { https://drive.google.com/drive/fol } \\
\text { ders/1WTYAja8R30t- } \\
\text { 1Pf7kX9h6hXvclwozv6d } \\
\text { - Cómo se despliegan los } \\
\text { contenidos } \\
\text { Cuando un/a espectador/a }\end{array}$ \\
\hline
\end{tabular}


el monitor de la zona 1 y en la proyección de la zona 2 se reproduce el video del evento seleccionado. Sobre la interface aparecen diferentes fechas, al clicar sobre alguna de ellas se muestra la imagen de un artículo de prensa sobre ese evento correspondiente a esa fecha. Si durante 2 minutos nadie interactúa, en el monitor de la zona 1 y en la proyección de la zona 2 se reproduce un video con los nombres de los miembros de la paltaforma Salvem el Cabanyal en scroll de abajo a arriba.

- Historia de la producción y personas implicadas:

Coordinación: Emilio Martínez y Maribel Domènech

Grabación video: Francisco Sanmartín.

Cámara: Sony DCR-1000E Camcorder DV-PAL Diseño interface gráfica: Moisés Mañas

Software: Director (Macromedia)

Edición video: María José Martínez de Pisón y Trinidad Gracia

Software: Premiere 6 (Adobe)

Hardware: Matrox DSDTI/1 Digimix Digisuite Video Capture Editing $\mathrm{PCl}$ selecciona alguna de las 5 opciones que muestra la interface, en la proyección de la zona 2 se reproduce el video del evento seleccionado. Sobre la interface aparecen diferentes fechas, al clicar sobre alguna de ellas se muestra la imagen de un artículo de prensa sobre ese evento correspondiente a esa fecha. $\mathrm{Si}$ durante 2 minutos nadie interactúa, en la proyección de la zona 2 se reproduce al azar uno de los videos de la conversación entre vecinos, al terminar, si nadie interactúa, se reproduce un video con los nombres de los miembros de la paltaforma Salvem el Cabanyal en scroll de abajo a arriba.

- Historia de la producción y personas implicadas Migración de archivos, código e interface: Emanuele Mazza Exportación archivos video con FFmpeg 
Entre_Cabanyal upgrade. Versiones y actualizaciones en las obras de arte digital interactivo

\begin{tabular}{|l|c|}
\hline $\begin{array}{l}\text { Imágenes: Moisés Mañas } \\
\text { Software: Photoshop } 5.0 \text { (Adobe) }\end{array}$ & \\
\hline Valoración de su estado & \\
\hline $\begin{array}{c}\text { Ya no es posible instalarla en un ordenador. } \\
\text { Todos los componentes digitales de la obra } \\
\text { están guardados en soporte CD-ROM. }\end{array}$ & $\begin{array}{c}\text { Estado correcto. Se ha actualizado } \\
\text { la obra con nuevos dispositivos y } \\
\text { código }\end{array}$ \\
\hline
\end{tabular}

Tabla 1. Comparación datos técnicos Entre_Cabanyal, versión original y actualización Fuente: Los autores (2021)

\section{Conclusiones}

El desarrollo de contenidos académicos y de investigación sobre la preservación de las obras de arte digital interactivo que se ha producido en los últimos años nos ha hecho mucho más conscientes de que tenemos que documentar con rigor las obras y que, mientras nuestra memoria siga activa, junto al empuje de generar nuevas experiencias artísticas, tenemos que procurar su durabilidad e ir actualizando las obras anteriores con una documentación pormenorizada de cada proceso. La documentación detallada de los componentes de la obra nos ha permitido decidir los cambios técnicos necesarios con una mayor comprensión en su conjunto.

Ante la pregunta inicial: ¿Al actualizar estas obras podemos considerarlas como versiones o como nuevas obras? Toda instalación artística que no sea site-specific, al ser montada en espacios diferentes se ve condicionada por las características de cada uno de los espacios, y además, si se presenta en una muestra colectiva, como es el caso de esta última exposición de la obra, se ve influenciada también por las otras obras próximas. De ahí que podamos concluir, que las instalaciones interactivas pueden ser consideradas siempre como versiones, más allá de los cambios técnicos en sus dispositivos y programación que se haya realizado para su preservación.

\section{Referencias}

Benjamin, W. (1996). La tarea del traductor. En Teorías de la traducción. Antología de textos (Dámaso López García). Ediciones de la Universidad de Castilla-La Mancha.

COPESTAKE, J. (2013). Saving digital art from an early death at the ZKM museum [BBC]. BBC. https://www.bbc.com/news/technology-24585267

Digital Art Conservation. (s.f.). [Jeffery Shaw, The Legible City, 1989-1991]. Digital Art Conservation. Recuperado 18 de abril de 2021, de 
http://www.digitalartconservation.org/index.php/en/exhibitions/zkmexhibition/nnnnnjeffrey-shaw.html

Escobedo, K. (2011). Smithsonian Institution Archives [Digital Video Preservation: Further Challenges for Preserving Digital Video and Beyond]. Smithsonian Institution Archives. https://siarchives.si.edu/blog/digital-video-preservation-further-challenges-preservingdigital-video-and-beyond

Garcia, L., \& Montero Vilar, P. (2010). The Challenges of Digital Art Presentation. e-conservation, 43-53.

LABORATORIO DE LUz. (s. f.). Laboratorio de Luz Entre_Cabanyal [Entre_Cabanyal]. Recuperado 21 de marzo de 2020, de http://laboluz.webs.upv.es/projects/entre_cabanyal/

MARCHAN FIZ, S. (1994). Del arte objetual al arte de concepto. Akal.

MATters in Media ART. (2015). [Documenting Media Art]. Matters in Media Art. http://mattersinmediaart.org/downloads/mattersmediaart_structureconditionreport_c omputuerworksprompt.rtf

MUÑOz VIÑAS, S. (2010). Teoría contemporánea de la Restauración. Síntesis.

NoBlE, J. (2009). Programming Interactivity. A Designer's Guide to Processing, Arduino, and openFrameworks. O'Reilly.

Plataforma Salvem el Cabanyal. (s. f.). Salvem el Cabanyal-la Resistència [Salvem el Cabanyal]. Recuperado 18 de abril de 2021, de http://cabanyal.com/els-fets/

VENTURINI, S., \& SANTI, M. (2013). The history and technological characteristics of cinematographic production and reception devices. En Preserving and exhibiting media art. Challenges and Perspectives (Noordegraaf, Julia; Saba , Cosetta G., Le Maître, Barbara, Hediger, Vinzenz). Amsterdam University Press. 